\title{
Utilisation de la PSPB pour le diagnostic de gestation des ruminants
}

\author{
P Humblot
}

UNCEIA, laboratoire de dosages hormonaux, 13, rue Jouët, BP 65, 94703 Maisons-Alfort, France

(Received 26 September 1991; accepted 6 January 1992)

\begin{abstract}
Résumé - La PSPB (Pregnancy specific protein B) a été isolée initialement à partir de membranes placentaires chez la vache. Il a été montré que cette protéine est le premier signal embryonnaire détectable dans la circulation périphérique maternelle. Le développement d'un système de dosage radio-immunologique spécifique a permis d'étudier les profils de cette protéine pendant la gestation dans plusieurs espèces de ruminants (ie, vache, brebis, chèvre, biche) et plus particulièrement chez la vache dans les cas de mortalité embryonnaire ou d'avortement. Dans cet article sont présentées les données obtenues avec ce dosage chez la vache, la chèvre et les cervidés, pendant le postpartum et le début de la gestation. Les résultats d'exactitude lorsque cette méthode est utilisée à des fins de diagnostic de gestation sont également décrits.
\end{abstract}

gestation / PSPB / diagnostic / ruminant / embryon / protéine

Summary - Use of PSPB radioimmunoassay (RIA) for pregnancy diagnosis in ruminants. Pregnancy specific protein B (PSPB) has been isolated from placental membranes of the cow and sheep. It has been shown that this protein was the first embryonic signal detectable in the matemal circulation. Development of a specific radioimmunoassay (RIA) for PSPB has allowed profiles of this protein to be studied during the pregnancy of many ruminants (ie cow, ewe, goat, mountain goat, cervids) or especially in the cow in case of embryonic mortality or abortion. This paper reviews data obtained with the PSPB RIA in the cow, goat and deer during the post-partum period and establishment of pregnancy. The accuracy of the results of PSPB RIA when used as a method of pregnancy diagnosis has been examined.

pregnancy / PSPB / diagnosis / ruminant / embryo

\section{INTRODUCTION}

L'existence d'une protéine de gestation détectable dans la circulation périphérique maternelle; la PSPB (pregnancy specific protein B) a été rapportée pour la première fois chez la vache par Butler et al
(1982). Cette molécule d'origine trophoblastique, mais différente par ses caractéristiques physicochimiques des protéines impliquées dans le processus antilutéolytique, a été isolée à partir d'embryons âgés de 25-45 j. Des protéines voisines ont été isolées chez la vache (Beckers et al, 
1988; Camous et al, 1988) et plus récemment chez la brebis (Ruder et al, 1988; Camous et al, 1990). Le(s) rôle(s) de la PSPB pendant la gestation reste(nt) mal connu(s), cependant certaines propriétés immunosuppressives ont été mises en évidence par Dunbar et al (1990).

Le dosage radio-immunologique de la PSPB dans le sérum ou le plasma maternel, initialement mis au point et décrit chez les bovins par Sasser et al (1986) a été utilisé ou adapté dans d'autres espèces de ruminants. Cet article présente les concentrations plasmatiques obtenues avec ce dosage au cours du post-partum, du début de la gestation, ou à l'occasion de mortalité embryonnaire chez les bovins, les petits ruminants et les cervidés, ainsi que les données d'exactitude observées lorsque cette méthode de dosage est utilisée à des fins de diagnostic de gestation.

\section{ÉVOLUTION DES CONCENTRATIONS DE PSPB AU COURS DU POST-PARTUM}

Chez les bovins, l'existence de concentrations de protéine très élevées (500-800ng/ $\mathrm{ml}$ ) au moment de la parturition associée à la longue demi-vie $(7 \mathrm{j})$ de la molécule (Ruder et Sasser, 1986) provoquent le maintien de concentrations sériques ou plasmatiques détectables pendant environ $100 \mathrm{j}$ chez la vache laitière (Humblot et al, 1988a) comme chez la vache allaitante (Humblot et al, 1990a; fig 1).

Chez la chèvre, la régression des concentrations au cours du post-partum est complète dans les 50 j qui suivent la mise bas (Humblot et al, 1990b).

Chez le cerf rouge, avec le système de dosage bovin (anticorps anti-bPSPB et protéine marquée bovine), les concentrations sériques au moment de la mise-bas sont comprises entre 5 et $10 \mathrm{ng} / \mathrm{ml}$. Ces concentrations, semblables à celles décrites par Fisher et al (1986) chez les cervidés mais relativement faibles par rapport à celles observées chez la vache et la chèvre expliquent en partie la disparition très rapide de la PSPB au cours du post partum dans cette espèce (fig 2).

\section{CONCENTRATIONS DE PSPB AU DÉBUT DE LA GESTATION ET EN CAS DE MORTALITÉ EMBRYONNAIRE}

Chez les bovins, les concentrations sériques ou plasmatiques de PSPB augmen-

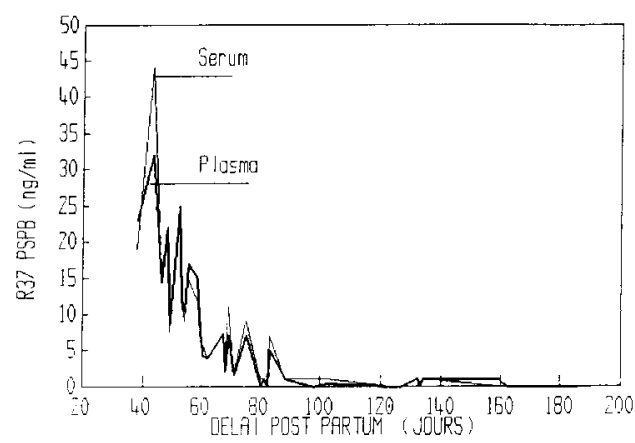

Fig 1. Concentrations de PSPB dans le serum et dans le plasma de vaches allaitantes au cours du post partum (seion Humblot et al, 1990a).

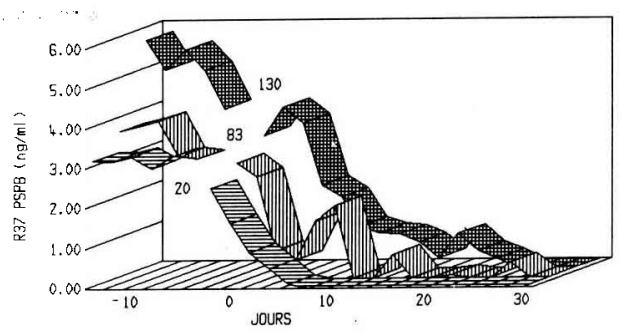

Fig 2. Concentrations de PSPB mesurées dans le serum de biches (cerf rusa) au cours du post partum (Bianchi et Humblot, non publié). 
tent entre le $20^{\mathrm{e}}$ et le $30^{\mathrm{e}} \mathrm{j}$ de gestation (Sasser et al, 1986; Humblot et al, 1988b). La PSPB est donc le premier signal embryonnaire détectable dans la circulation périphérique des femelles gestantes. Cependant, le moment auquel la PSPB peut être mise en évidence pour la première fois varie beaucoup d'un individu à l'autre (Humblot, 1988). Tous les animaux gestants présentent une concentration détectable au $30^{\mathrm{e}} \mathrm{j}$ de gestation. Celle-ci augmente ensuite jusqu'à la parturition pour atteindre parfois des valeurs supérieures à $700 \mathrm{ng} / \mathrm{ml}$.

En cas d'absence de fécondation ou de mortalité embryonnaire précoce avant le $16 \mathrm{e}$ j (lorsque la sécrétion de progestérone n'est pas maintenue au-delà de $21 \mathrm{j}$ ), il est tout à fait exceptionnel de retrouver des concentrations de PSPB détectables au $30^{\circ}$ j de gestation présumée (tableau I). En effet, chez les vaches laitières comme chez les vaches allaitantes, la fréquence de cette situation est égale à $3 \%$.
La mortalité embryonnaire tardive (lorsque les animaux présentent une concentration de progestérone élevée 21-24 j après insémination mais sont ultérieurement non gestants) peut représenter selon les troupeaux 8-20\% du total des vaches inséminées. Dans ce groupe, la proportion des animaux chez lesquels la PSPB est détectable est relativement faible et varie de 20 à $30 \%$ des cas de mortalité embryonnaire tardive estimés par dosage de progestérone (tableau I). La fréquence de ces cas est indépendante du taux de fertilité global de l'échantillon. Enfin lorsque la PSPB est détectable, les concentrations moyennes observées chez les femelles gestantes sont plus élevées que celles des vaches qui présentent une mortalité embryonnaire tardive (Humblot et al, 1988b). Cependant, la variabilité des concentrations d'un animal à l'autre est telle qu'il n'est généralement pas possible de prévoir avec certitude et individuellement l'arrêt de la gestation dès le $30^{\mathrm{e}} \mathrm{j}$ qui suit la concep-

Tableau I. Fréquence des échecs de gestation et des animaux présentant une élévation des concentrations plasmatiques de PSPB $\left(\mathrm{PB}+\right.$ ) entre le $28^{\mathrm{e}}$ et le $30^{\mathrm{e}} \mathrm{j}$ de gestation (selon Humblot et al, 1988a, b, d; 1990a).

\begin{tabular}{|c|c|c|c|c|c|c|}
\hline \multirow[b]{3}{*}{$\begin{array}{l}\text { Situation } \\
\text { après IA }\end{array}$} & \multicolumn{6}{|c|}{ Expériences } \\
\hline & \multicolumn{2}{|c|}{ Humblot et al (1988b) } & \multicolumn{2}{|c|}{ Humblot et al $(1988 a, d)$} & \multicolumn{2}{|c|}{ Humblot et al (1990a) } \\
\hline & \multicolumn{2}{|c|}{ Race FFPN } & \multicolumn{2}{|c|}{ Race normande } & \multicolumn{2}{|c|}{ Races allaitantes } \\
\hline $\begin{array}{l}\text { Non fécondation } \\
\text { or mortalité }\end{array}$ & 63 & 35,6 & 44 & 37 & 53 & 39 \\
\hline $\begin{array}{l}\text { embryonnaire } \\
\text { précoce }\end{array}$ & $\mathrm{PB}+$ & $\begin{array}{l}3,1 \\
(2 / 63)\end{array}$ & $\mathrm{PB}+$ & $\begin{array}{l}2,3 \\
(1 / 44)\end{array}$ & PB+ & $\begin{array}{l}1,9 \\
(1 / 53)\end{array}$ \\
\hline $\begin{array}{l}\text { Mortalité } \\
\text { embryonnaire } \\
\text { tardive }\end{array}$ & $\begin{array}{l}31 \\
\mathrm{~PB}+\end{array}$ & $\begin{array}{l}17,5 \\
32,3 \\
(10 / 31)\end{array}$ & $\begin{array}{l}10 \\
\text { PB+ }\end{array}$ & $\begin{array}{l}8,4 \\
30 \\
(3 / 10)\end{array}$ & $\begin{array}{l}19 \\
\mathrm{~PB}+\end{array}$ & $\begin{array}{l}14 \\
21 \\
(4 / 19)\end{array}$ \\
\hline Gestation & 83 & 46,9 & 65 & 54,6 & 64 & 47 \\
\hline Nombre total & 177 & 100 & 119 & 100 & 136 & 100 \\
\hline
\end{tabular}


tion (Humblot et al, 1988b). Dans ces cas d'avortement, des données préliminaires obtenues après induction d'avortement à l'aide de prostaglandines, montrent que l'élimination de la PSPB est relativement rapide (fig 3). En effet les concentrations à $40 \mathrm{j}$, voisines de $2 \mathrm{ng} / \mathrm{ml}$, deviennent non détectables près de 10 jours après l'injection de prostaglandines. Ceci confirme les données obtenues par Semanbo et al (1990), après induction d'avortement à ce stade de gestation par cette même méthode ou inoculation d'Actinomycès Pyogenes.

La préparation d'un anticorps dirigé contre la PSPB ovine (Ruder et al, 1988) a permis d'obtenir un système sensible pour doser cette protéine chez les ovins (Ruder et al, 1988) et les caprins (Humblot et al, 1990b). Chez la chèvre alpine, dans un système de dosage radio-immunologique hétérologue (protéine bovine pour le standard et la protéine marquée; anticorps anti PSPB ovine), les concentrations de PSPB augmentent comme chez les bovins entre le $20^{\mathrm{e}}$ et le $30^{\mathrm{e}} \mathrm{j}$ de gestation. Bien que les concentrations moyennes de cette protéine soient supérieures en cas de gestation gémellaire ou triple à celles observées en cas de gestation simple, les variations individuelles très importantes ne permettent pas d'utiliser cette méthode à des fins

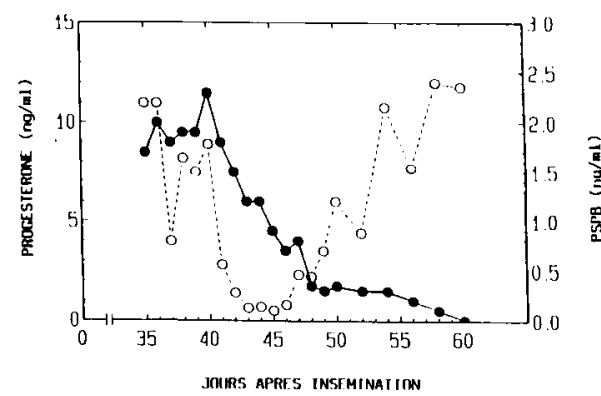

Fig 3. Concentrations de Progesterone et de PSPB après induction d'avortement par une injection de prostaglandines au 40 e jour de gestation (selon Humblot et Gary, non publié). de diagnostic de gémellité (Humblot et al, 1990b).

En cas de mortalité embryonnaire tardive (progestérone élevée à $21 \mathrm{j}$ et $\mathrm{ab}$ sence de mise-bas) et comme chez la vache, la PSPB peut être ou ne pas être détectée aux premiers stades de la gestation. Ceci a été montré à partir d'un effectif de près de 200 chèvres $(n=189)$ diagnostiquées positives à 21 j par dosage de progestérone dans la matière grasse du lait. Sur cet ensemble le taux de femelles gestantes à $70 \mathrm{j}$ a été de $75 \%$ (141/189). Parmi les animaux ayant avorté entre 21 et $70 \mathrm{j}(n=48)$, moins de la moitié ont présenté des concentrations de PSPB détectables vers $30 \mathrm{j}(20 / 48)$. Chez ces femelles (fig 4), les concentrations moyennes de PSPB observées entre 30 et 40 j sont plus faibles que celles des chèvres encore gestantes à $70 \mathrm{j}(1,8 \pm 0,1$ vs $4,9 \pm 0,28 \mathrm{ng} / \mathrm{ml}$; $P<0,001)$.

Lors de pseudogestation (image échographique révélant une augmentation de volume de l'utérus associé à une accumulation de liquide sans fœtus détectable), l'absence de sécrétion de PSPB initialement montrée pour une femelle chez la-

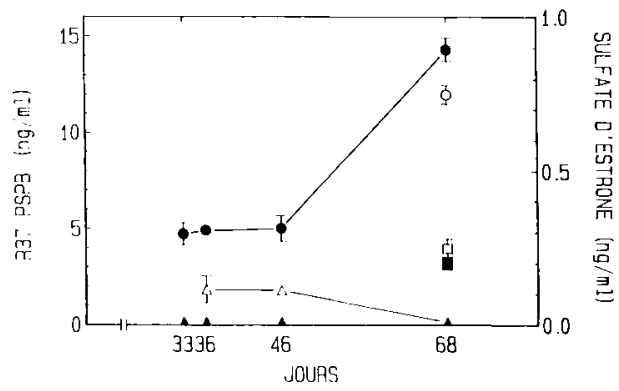

Fig 4. Concentrations de PSPB entre le $33^{e}$ et le $68^{\mathrm{e}}$ jour et de sulfate d'œstrone au $68^{\mathrm{e}}$ jour après l'insémination chez des chèvres gestantes (@); SE, O) ou ayant présenté un avortement embryonnaire après le $21^{\mathrm{e}}$ jour $(\Delta-\Delta$ et $\Delta-\Delta ;$ SE $\square$ et $\mathbf{D}$ ) (selon Humblot $e$ t $a$, non publié). 
quelle des prélèvements fréquents étaient effectués (Humblot et al, 1990b) a été vérifiée sur plus d'une centaine de chèvres pseudo-gestantes (Duquesnel et al, 1992). Ceci montre bien, qu'en dépit du maintien systématique de concentrations de progestérone élevées, ce processus est indépendant de l'installation d'une gestation.

Chez les cervidés, la réalisation de prélèvements fréquents est souvent problématique et rend difficile la détermination d'un profil moyen au début de la gestation. Cependant à partir de prélèvements ponctuels, Fisher et al (1986), ont obseivé, comme chez les autres ruminants, une augmentation des concentrations vers le $30^{\mathrm{e}} \mathrm{j}$ de gestation. Toutefois, avec le système de dosage «bovin» les concentrations au cours de la gestation apparaissent plus faibles que chez la vache et sont toujours inférieures à $1 \mathrm{ng} / \mathrm{ml}$ entre 30 et $40 \mathrm{j}$.

\section{UTILISATION DU DOSAGE DE PSPB POUR LE DIAGNOSTIC DE GESTATION}

Ce dosage a été utilisé comme méthode de diagnostic de gestation essentiellement chez les ruminants domestiques.
Chez les bovins, le faible nombre d'animaux chez lesquels la PSPB augmente mais qui ne vêlent pas, ainsi que l'élimination relativement rapide de la protéine dans la circulation périphérique après avortement embryonnaire, expliquent pourquoi dans les conditions expérimentales (Humblot et al, 1988c, d) ou de routine (tableau II), l'exactitude des résultats positifs après le $30^{\mathrm{e}} \mathrm{j}$ est très bonne (voisine de $90 \%$ ) et toujours supérieure à celle du dosage de progestérone à $21 \mathrm{j}$ postinsémination (comprise entre 70 et $75 \%$ ), lorsque les échantillons sont prélevés et les analyses réalisées chez les mêmes animaux.

L'exactitude des résultats négatifs (nombre de femelles déclarées non gestantes et réellement non gestantes/ nombre total de femelles déclarées non gestantes) ne diffère pas d'une méthode à l'autre et est toujours proche de $100 \%$. Enfin la détection précoce des femelles non gestantes en fin de campagne est meilleure avec le dosage de PSPB (85\% environ) qu'avec le dosage de progestérone (58\%). Ainsi, le dosage de PSPB apparaît plus efficace que le dosage de progestérone dans le contrôle de la

Tableau II. Exactitude des diagnostics de gestation par dosage de PSPB, plus de 30 jours après IA. Comparaison avec celles du dosage de progestérone à 21 jours (P4) lorsque les 2 analyses ont été effectuées chez les mêmes animaux.

\begin{tabular}{|c|c|c|c|}
\hline \multirow[t]{2}{*}{ Méthode } & Exactitude + & Exactitude - & $\begin{array}{l}\text { Détection des femelles } \\
\text { non gestantes }\end{array}$ \\
\hline & (\%) & $(\%)$ & (\%) N \\
\hline $\begin{array}{l}\text { Résultats de } \\
\text { dosage de PSPB } \\
\text { contrôlés } \\
(N=1461)\end{array}$ & $94,3 \% \quad(935 / 991)$ & $99,5 \% \quad(446 / 448)$ & $86,3 \%(446 / 517)$ \\
\hline $\begin{array}{l}\text { Résultats des } \\
\text { dosages de PSPB } \\
\text { et progestérone } \\
\text { (P4) contrôlés } \\
(N=617)\end{array}$ & $\begin{array}{ll}88,3 \% & (295 / 334) \\
71,3 \% & (303 / 425)\end{array}$ & $\begin{array}{l}98,5 \% \\
9761 / 265) \\
97,8 \%(177 / 181)\end{array}$ & $\begin{array}{l}85,3 \%(261 / 306) \\
57,8 \%(177 / 306)\end{array}$ \\
\hline
\end{tabular}


reproduction du troupeau. C'est aussi un outil plus souple, car la contrainte de faire le prélèvement 21-24 j après l'IA n'existe pas. Cependant, malgré ces avantages, il est nécessaire de respecter scrupuleusement l'attente du $30^{\mathrm{e}} \mathrm{j}$ après IA pour effectuer le dosage. Des prélèvements plus précoces provoquent l'obtention de faux résultats négatifs car quelques vaches gestantes présentent une augmentation tardive des concentrations de PSPB. Bien que le nombre de femelles concernées soit très faible, la gravité d'interventions pouvant conduire à l'arrêt de la gestation amène à réaliser des travaux supplémentaires pour cerner les "populations à risque".

Par ailleurs, comme cela a été montré précédemment, dans toutes les études réalisées avec le dosage de cette protéine, des concentrations très élevées et persistant longtemps (jusqu'à 70-90 j) ont été observées au cours du post-partum. Ceci impose d'attendre le $100^{\mathrm{e}} \mathrm{j}$ post-partum pour réaliser les prélèvements. Dans le cas contraire, l'exactitude des résultats positifs est diminuée d'environ 10-15\% (Hum- blot et al, 1988a, 1990a). Lorsque ces 2 conditions, portant sur le délai de prélèvement, sont respectées, les qualités de la méthode permettent d'obtenir une véritable confirmation de la gestation. En outre, la souplesse d'utilisation en fait un outil très bien adapté à l'élevage allaitant.

Cette méthode de constat de gestation est apparue assez performante chez les petits ruminants (tableau III). On remarque cependant dans l'étude effectuée chez la brebis le faible nombre d'animaux prélevés à des stades précoces de gestation (avant 40 j). Les données obtenues chez les caprins dans 2 essais différents sont encourageantes. Cependant celles-ci illustrent les limites de la méthode lorsqu'elle est utilisée précocement. En effet avec des prélèvements réalisés vers le $26^{e} \mathrm{j}$, l'exactitude des résultats positifs ne diffère pas de celle du dosage de progestérone à 21 j réalisé chez les mêmes animaux $(79 \%$ vs $82 \%$ ). En revanche, lorsque des prélèvements (réalisés dans 7 troupeaux différents) sont effectués au stade $35-45 \mathrm{j}$, l'exactitude des résultats positifs du dosage de PSPB est supérieure à celle du

Tableau III. Exactitude du dosage de PSPB (réalisé à des fins de diagnostic de gestation chez les ovins, selon Ruder et al (1988) et les caprins (selon (2) Humblot (1988), (3) Humblot et al, non publié).

\begin{tabular}{llllll}
\hline Espèce & Essai & Délais & \multicolumn{3}{c}{ Exactitude des diagnostics } \\
post IA & ou saillie & $\%$ & Positifs & $\%$ & $\%^{\text {Négatifs }}$ \\
& & $\%$ & $n$ & $n$ \\
\hline
\end{tabular}

Brebis

(1)

$\begin{array}{ll}26-96 & 93,8 \\ 35-106 & 98,8 \\ 56-126 & 98,8 \\ 83-153 & 100\end{array}$

$(30 / 32)$
$(159 / 161)$
$(30 / 31)$
$(30 / 30)$

$\begin{array}{lll}100 & (30 / 30) & 100\end{array}$

Chèvre

$\begin{array}{llllll}(2) & 26 & 79,3 & (23 / 29) & 100 & (5 / 5) \\ (3) & 35-45 & 90,5 & (134 / 148) & 100 & (26 / 26)\end{array}$


dosage de progestérone $(91 \%$ vs $71 \%$, $P<0,05)$. De telles différences dans les taux d'exactitude ont été observées particulièrement dans 4 troupeaux sur les 7 étudiés. Dans 3 troupeaux dans lesquels on peut supposer une très faible fréquence d'avortements embryonnaires tardifs, le taux d'exactitude à été très comparable pour les 2 méthodes.

Ceci souligne que chez les petits ruminants, l'intérêt du dosage de PSPB dépend du taux d'avortements embryonnaires tardifs ou fœtaux enregistrés dans les troupeaux. Ce dosage apparaît, de la même façon que chez les bovins, comme une bonne méthode pour mener des investigations sur les avortements survenant plus de 25 j après la conception.

\section{RÉFÉRENCES}

Beckers JF, Wouters Ballman P, Ectors F (1988) Isolation and radioimmunoassay of a bovine pregnancy specific protein. Theriogenology 29, 219 (abstr)

Butler JE, Hamilton WC, Sasser RG, Ruder CA, Hass GM, Williams $J J$ (1982) Detection and partial characterization of two bovine pregnancy specific proteins. Biol Reprod 26, 925933

Camous S, Charpigny G, Guillemot M, Martal J, Sasser RG (1988) Purification of one bovine pregnancy specific protein by high performance liquid chromatography (HPLC). In: Proc BARD workshop on matemal recognition of pregnancy and maintenance of the corpus luteum. Jerusalem, 20-24 Mars, abstr 2

Camous S, Coste V, Guillomot M, Martal J (1990) Demonstration of an ovine conceptus protein immunologically related to bovine pregnancy serum protein (PSP 60). In: Proc 3 d int ruminant reprod symp, Nice $25-28$ Mars, abs 17

Dunbar MM, Wong TS, Ruder-Montgomery CA, Chew BP, Sasser RG (1990) Partial characterization of the immuno-suppressive properties of pregnancy specific protein B (PSPB). Theriogenology 33, Abs 220
Duquesnel R, Pavisot D, Pivot G, Mialot JP, Saboureau L, Etienne $P$, Delaval J, Gueraud JM, Pregenre E, de Montigny $G$, Guerrault $P$, Perrin $G$, Humblot $P$, de Fontaubert $Y$, Chemineau $P$ (1992) La pseudogestation chez la chèvre. Ann Zootech 41, 407-415

Fisher MW, Ruder CA, Corson ID, Fennessy PF, Sasser RG (1986) Detection of pregnancy in the red deer hind (Cervus elaphus) using a radioimmunoassay for a bovine pregnancy specific protein. In: Proc soc stud fertil annu conf abstr 69

Humblot P (1988) Proteines spécifiques de la gestation chez les ruminants. Reprod Nutr Dév 28, 1753-1762

Humblot $P$, Oliveira $O$, Ruder $C A$, Jeanguyot $N$, Thibier M, Sasser RG (1988a) Progesterone and PSPB concentrations at time of $\mathrm{Al}$ and at the beginning of pregnancy in dairy cows. In: Proc BARD workshop on maternal recognition of pregnancy and maintenance of the corpus luteum. Jerusalem, 20-24 Mars, abstr 62

Humblot P, Camous S, Martal J, Charlerey J, Jeanguyot N, Thibier M, Sasser RG (1988b) Pregnancy specific protein $B$, progesterone concentrations and embryonic mortality during early pregnancy in dairy cows. J Reprod Fertil 83, 215-223

Humblot P, Camous S, Martal J, Charlerey J, Jeanguyot N, Thibier M, Sasser RG (1988c) Diagnosis of pregnancy by radioimmunoassay of a pregnancy specific protein in the plasma of dairy cows. Theriogenology 30 , 257-269

Humblot P, Jeanguyot N, Ruder CA, Leriche I, Thibier M, Sasser RG (1988d) Accuracy of pregnancy diagnosis by PSPB RIA in the plasma of dairy cows 28 days after Al. In: Proc 3rd int congr anim reprod and artif insem, Dublin (short communication) vol $2 p$ 94

Humblot $P$, Payen $B$, Jeanguyot $N$, Thibier $M$, Sasser RG (1990a) Progesterone and pregnancy specific protein $B$ concentrations in serum and plasma 28-30 days after $\mathrm{Al}$ and their relationship with embryonic mortality in French beef breeds. In: Proc 3rd int ruminant reprod symp, Nice 25-28 mars, abstr 21

Humblot $P$, de Montigny $G$, Jeanguyot $N$, Tetedoie $F$, Payen $B$, Thibier $M$, Sasser $R G$ (1990b) Pregnancy specific protein B and 
progesterone concentrations in French Alpine goats throughout gestation. I Reprod Fertil 89, 205-212

Ruder CA, Sasser RG (1986) Source of bovine pregnancy specific protein $B$ during the post-partum period and estimation of halflife of bPSPB. J Anim Sci 63 (suppl 1), 335, Abstr

Ruder CA, Sasser RG, Dahmen JJ, Stellflug JN (1988) Detection of pregnancy in sheep by radioimmunoassay of sera for pregnancy specific protein B. Theriogenology 29,905911
Sasser RG, Ruder CA, Ivani KA, Butler JE, Hamilton WC (1986) Detection of pregnancy by radioimmunoassay of a novel pregnancy specific protein in serum of cows and a profile of serum concentrations during gestation. Biol Reprod 35,936-942

Semanbo DK, Eckersall PD, Sasser RG, Ayliffe TR (1990) Pregnancy specific protein B (PSP-B) and progesterone in monitoring viability of the embryo in early pregnancy in the cow after experimental infection with Actinomyces pyogenes. J Reprod Fertil July 1990 , abstr Ser No 5, p 30 